\title{
Supplementary of
}

\section{Production of $\mathrm{N}_{2} \mathrm{O}_{5}$ and $\mathrm{CINO}_{2}$ in summer in urban Beijing, China}

Wei Zhou ${ }^{1,2 \#}$, Jian Zhao ${ }^{1,2 \#}$, Bin Ouyang ${ }^{3}$, Archit Mehra ${ }^{4}$, Weiqi Xu ${ }^{1,2}$, Yuying Wang ${ }^{5}$, Thomas J. Bannan $^{4}$, Stephen D. Worrall ${ }^{4, a}$, Michael Priestley ${ }^{4}$, Asan Bacak ${ }^{4}$, Qi Chen ${ }^{6}$, Conghui Xie ${ }^{1,2}$, Qingqing 5 Wang $^{1}$, Junfeng Wang ${ }^{7}$, Wei Du ${ }^{1,2}$, Yingjie Zhang ${ }^{1}$, Xinlei Ge ${ }^{7}$, Penglin Ye ${ }^{8,11}$, James D. Lee ${ }^{9}$, Pingqing $\mathrm{Fu}^{1,2}$, Zifa Wang ${ }^{1,2}$, Douglas Worsnop ${ }^{8}$, Roderic Jones ${ }^{3}$, Carl J. Percival ${ }^{4, b}$, Hugh Coe ${ }^{4}$, Yele Sun ${ }^{1,2,10}$

${ }^{1}$ State Key Laboratory of Atmospheric Boundary Layer Physics and Atmospheric Chemistry, Institute of Atmospheric Physics, Chinese Academy of Sciences, Beijing 100029, China

${ }^{2}$ University of Chinese Academy of Sciences, Beijing 100049, China

$10{ }^{3}$ Department of Chemistry, University of Cambridge, Cambridge CB2 1EW, UK

${ }^{4}$ Centre for Atmospheric Science, School of Earth, Atmospheric and Environmental Science, University of Manchester, Manchester M13 9PL, UK

${ }^{5}$ College of Global Change and Earth System Science, Beijing Normal University, Beijing 100875, China

${ }^{6}$ College of Environmental Sciences and Engineering, Peking University, Beijing 100871, China

$15{ }^{7}$ School of Environmental Science and Engineering, Nanjing University of Information Science and Technology, Nanjing 210044, China

${ }^{8}$ Aerodyne Research, Inc., Billerica, Massachusetts 01821, USA

${ }^{9}$ National Centre for Atmospheric Science, University of York, Heslington, York YO10 5DD, UK

${ }^{10}$ Center for Excellence in Regional Atmospheric Environment, Institute of Urban Environment, Chinese Academy of

20 Sciences, Xiamen 361021, China

${ }^{11}$ Nanjing DiLu Scientific Instrument Inc, Nanjing, 210036, China.

${ }^{a}$ Now at School of Materials University of Manchester, M13 9PL, UK

${ }^{b}$ Now at Jet Propulsion Laboratory, 4800 Oak Grove Drive, Pasadena, CA 91109

${ }^{\#}$ These authors contributed equally to this work

Correspondence: Yele Sun (sunyele@mail.iap.ac.cn) and Hugh Coe (hugh.coe@manchester.ac.uk) 

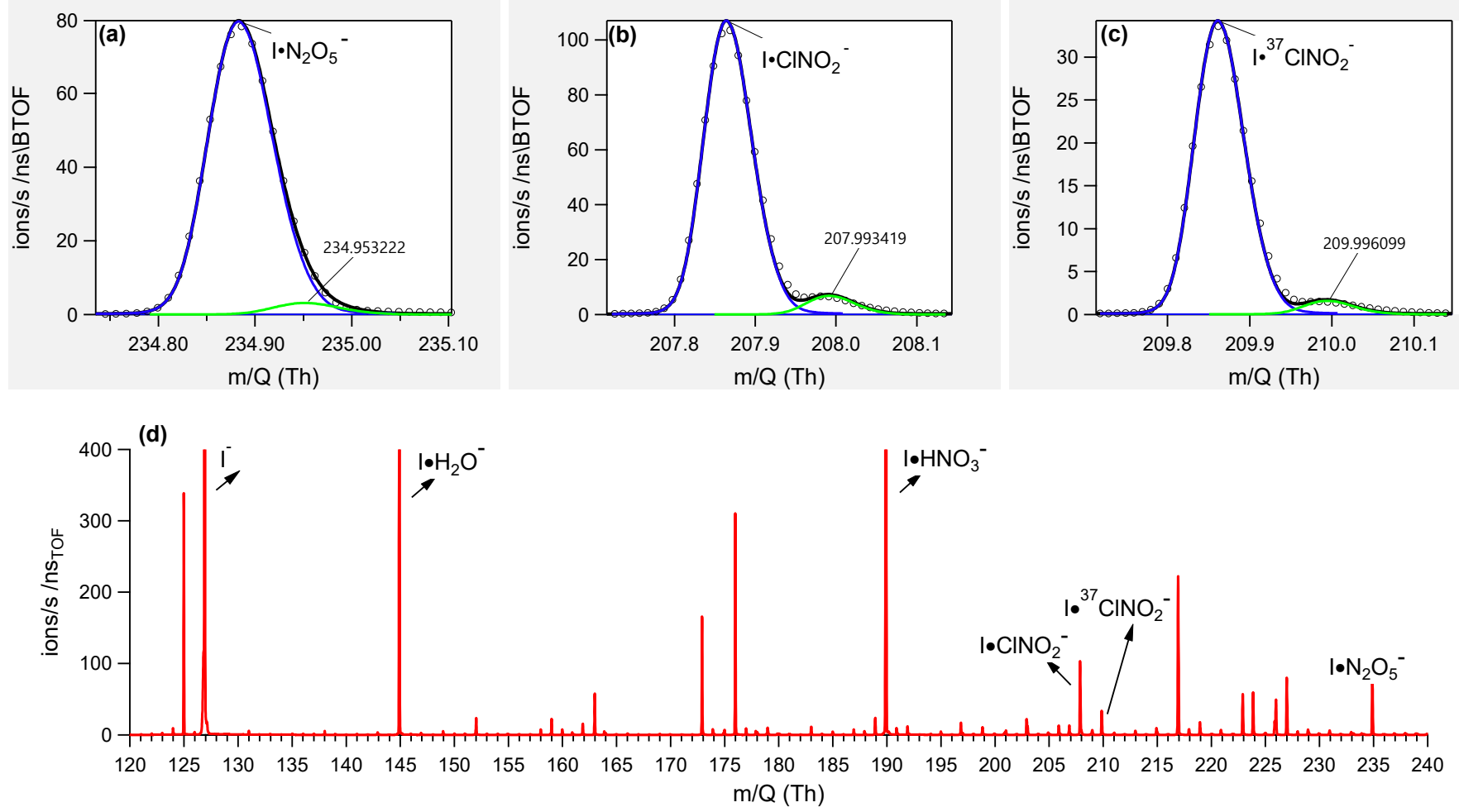

Figure S1. High resolution peak fitting for (a) $m / z 235$ as $\mathrm{I}^{\circ} \mathrm{N}_{2} \mathrm{O}_{5}{ }^{-}$, (b) $\mathrm{m} / \mathrm{z} 208$ as $\mathrm{I} \cdot \mathrm{ClNO}_{2}{ }^{-}$, (c) $m / z 210$ as $\mathrm{I}^{37} \mathrm{ClNO}_{2}{ }^{-}$, and (d) average high-resolution mass spectrum for one night from the IAP-CIMS measurement.

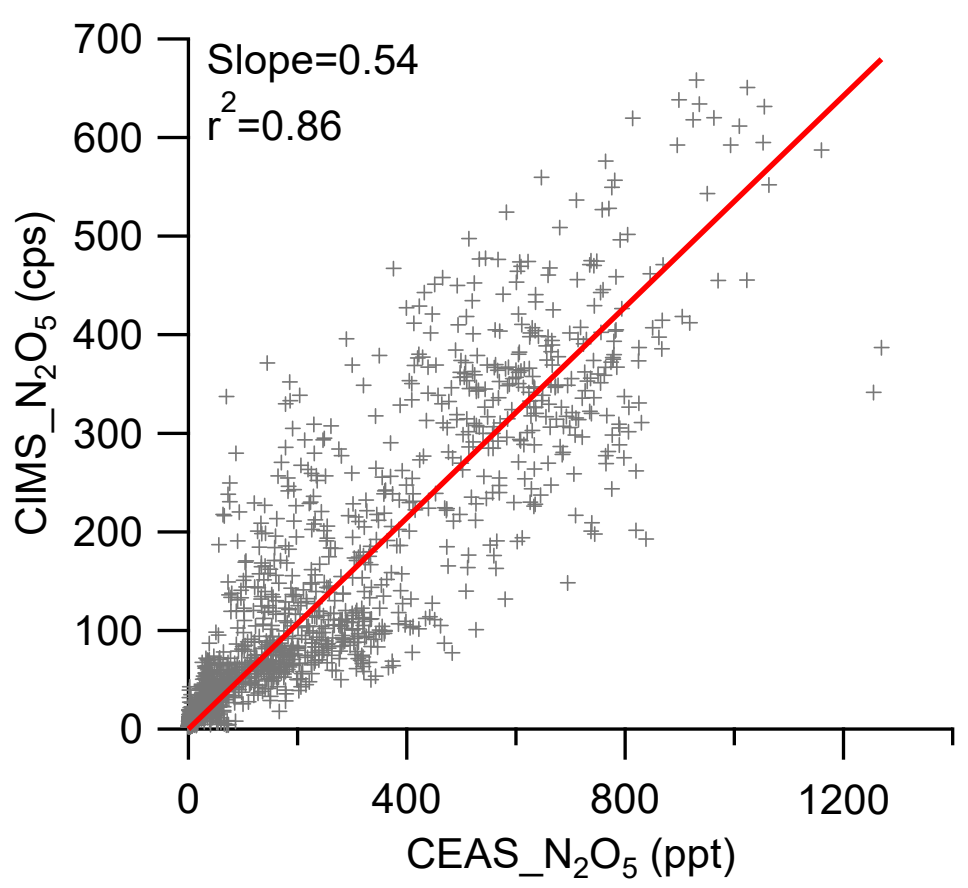

5 Figure S2. Comparison of IAP-CIMS raw signals of $\mathrm{N}_{2} \mathrm{O}_{5}$ with those measured by the Cambridge Broadband Cavity Enhanced Absorption Spectrometer (BBCEAS). The derived sensitivity of IAP-CIMS $\mathrm{N}_{2} \mathrm{O}_{5}$ is $0.54 \mathrm{cps} \mathrm{pptv}^{-1}$. 


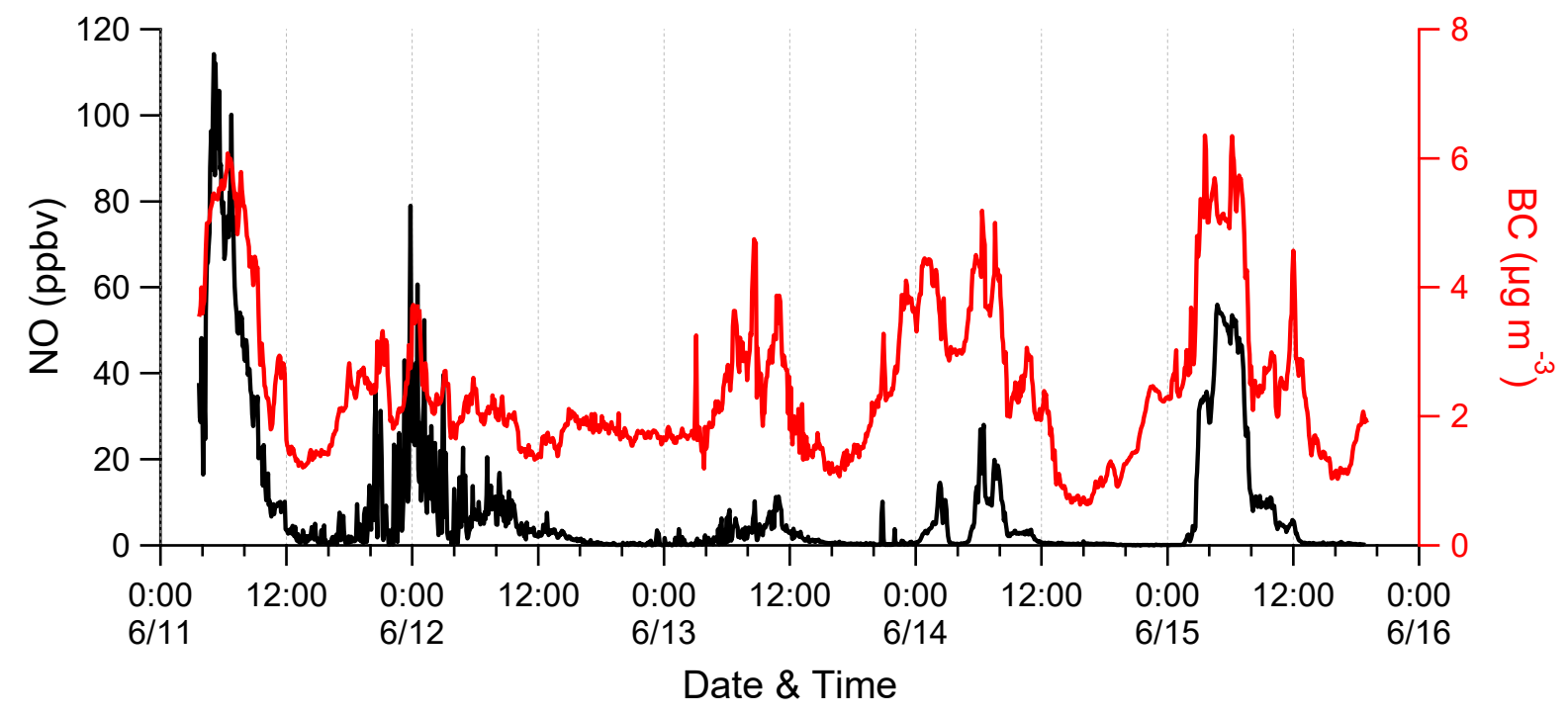

Figure S3. Time series of $\mathrm{NO}$ and $\mathrm{BC}$ during the campaign. 

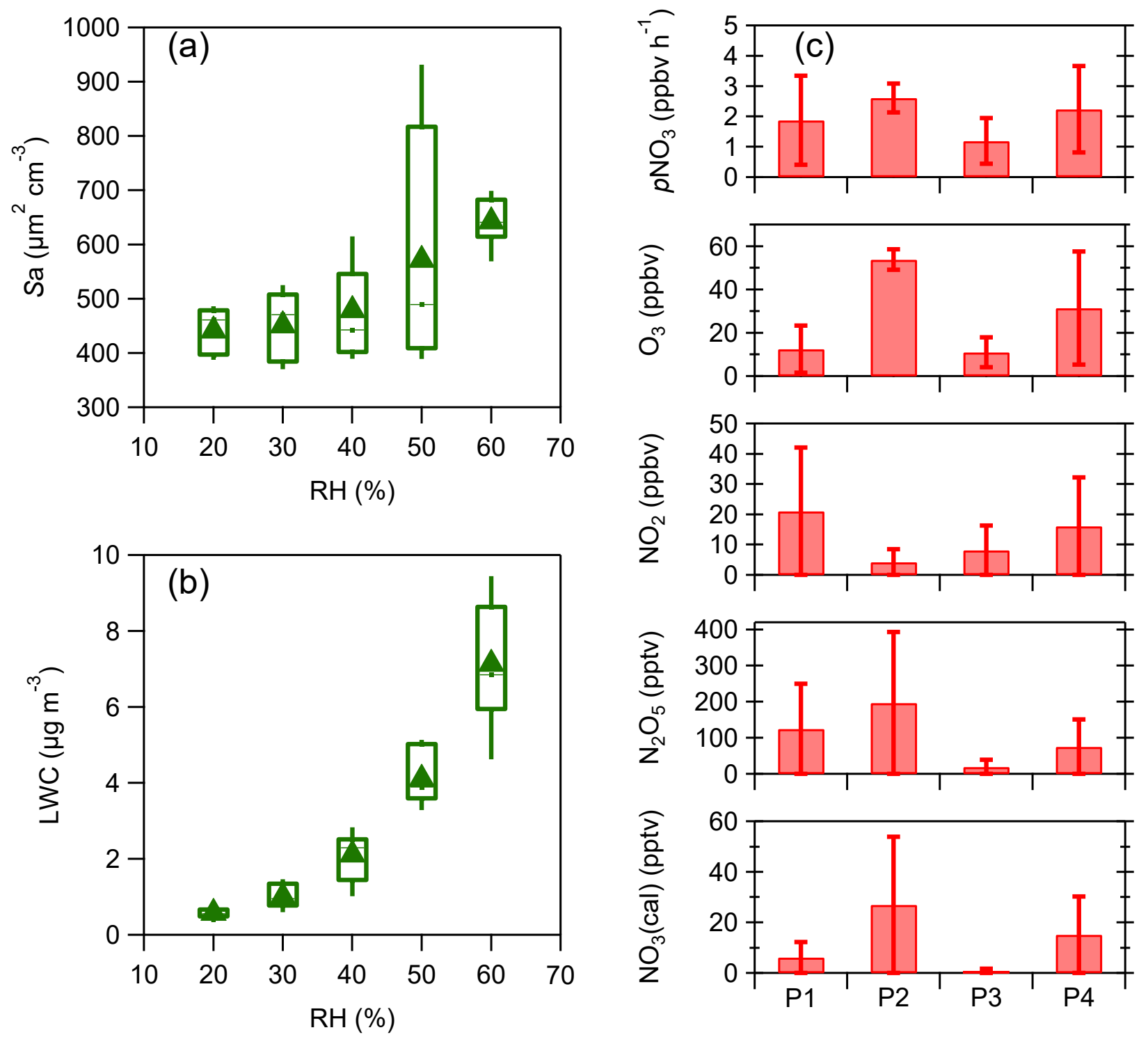

Figure S4. Variations of (a) aerosol surface area $\left(S_{\mathrm{a}}\right)$, and (b) aerosol liquid water content (LWC) as a function of RH. (c)

The average mixing ratios of and $\mathrm{NO}_{3}, \mathrm{~N}_{2} \mathrm{O}_{5}, \mathrm{NO}_{2}, \mathrm{O}_{3}$ and the nitrate radical production rate $p\left(\mathrm{NO}_{3}\right)$ for four different nights (i.e., P1, P2, P3 and P4). 

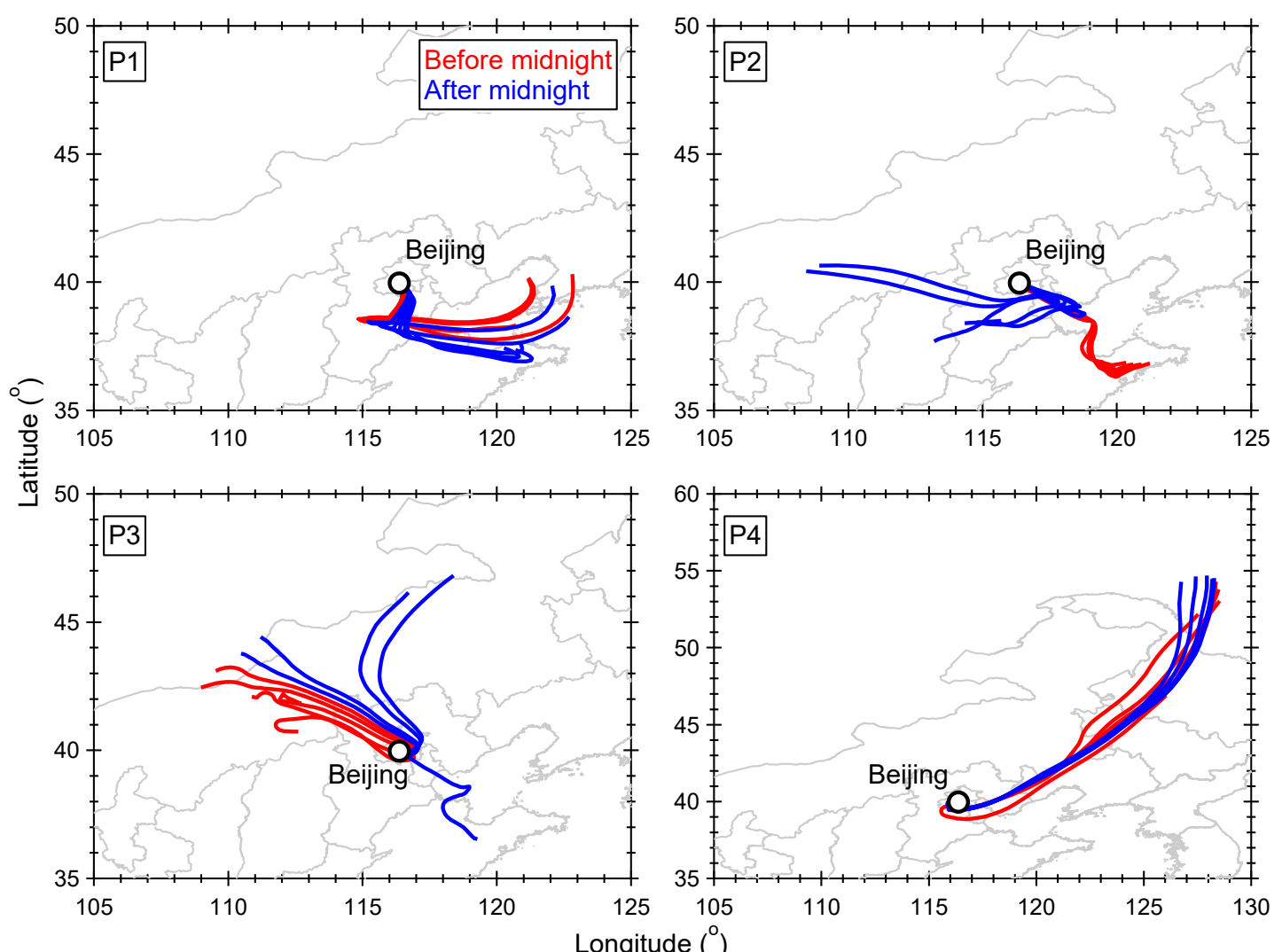

Figure S5. The 48-h back trajectories arrived at the sampling site during four different nights (i.e., P1, P2, P3 and P4). Note that the trajectories at each night are divided into two periods,i.e., before and after midnight. 


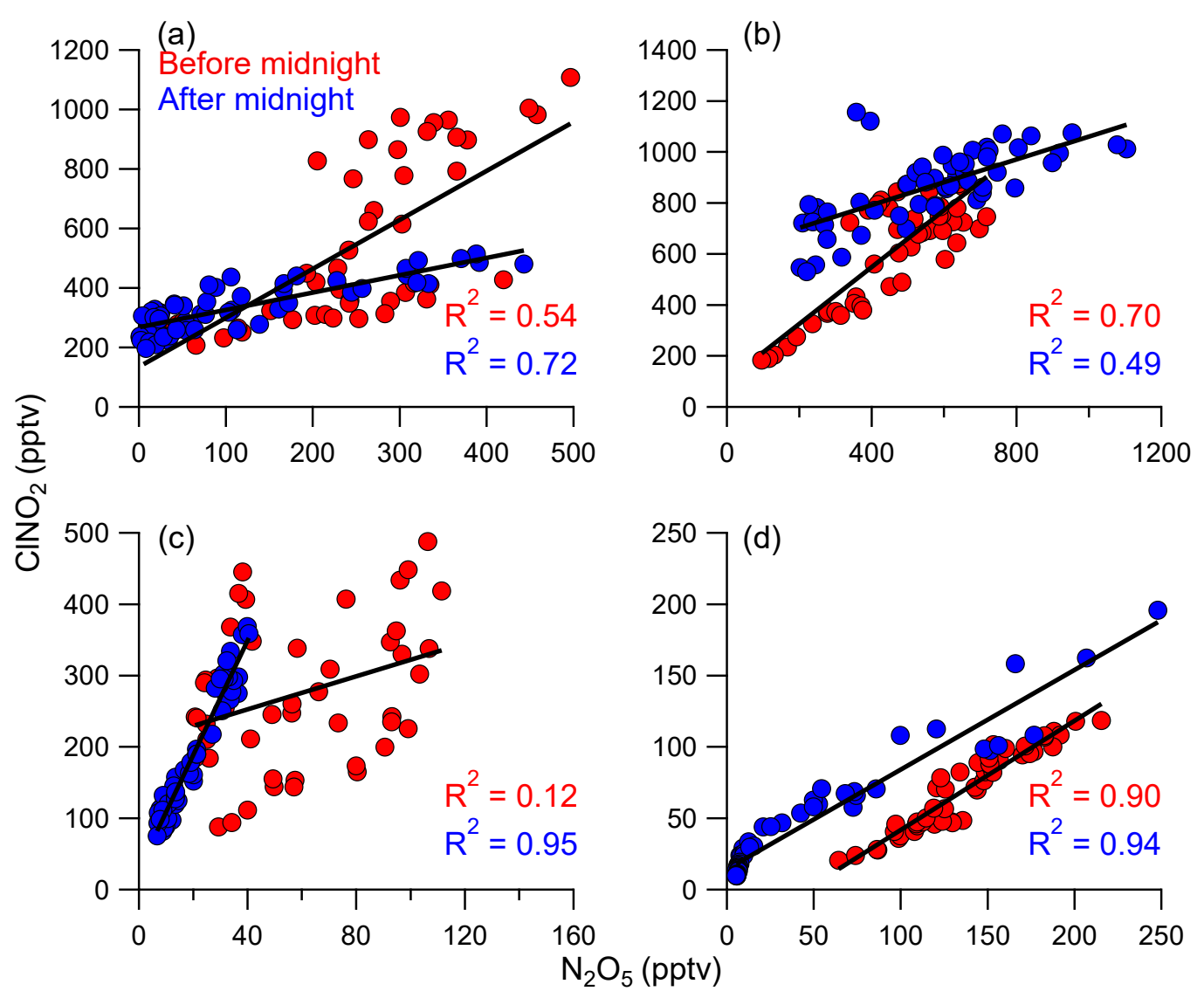

Figure S6. The correlations between $\mathrm{ClNO}_{2}$ and $\mathrm{N}_{2} \mathrm{O}_{5}$ for four different nights (i.e., P1, P2, P3 and P4). All the correlations are divided into two periods, i.e., before midnight and after midnight. 